phys. stat. sol. (b) 196, 85 (1996)

Subject classification: 72.90 and $73.61 ; 72.30 ; \mathrm{S} 7.12 ; \mathrm{S} 7.15$

Physico-Technical Institute, Academy of Sciences of Belarus, Minsk ${ }^{1}$ )

\title{
Beam Instability and Space-Charge Wave Amplification Caused by the Electron Injection out of QW into 2DEG
}

By

I. E. Tralle and V. A. Siz.JuK

(Received November 24, 1995; in revised form March 27, 1996)

The amplification of a space-charge wave in a two-dimensional electron gas induced by a beam instability is considered. The electron beam is regarded as being injected out of the quantum well by means of resonant tunneling. The beam-2DEG interaction is treated in terms of the hydrodynamic equations of a two-component liquid and the Poisson equation. The numerical solution of the dispersion equation is obtained, the increment of SCW is calculated and the dependence of the increment on the frequency tending to the 2D-plasmon frequency is considered.

Es wird dic Verstärkung von Raumladungswellen als Folge einer Instabilität des Elektronenstrahls in einem zweidimensionalen Elektronengas betrachtet. Der Elektronenstrahl wird mittels Resonanztunneln aus dem Quantentopf im 2DEG injiziert. Die Wechselwirkung des Strahls mit dem zweidimensionalen Elektronengas wird durch Gleichungen der Hydrodynamik für zwei Flüssigkeiten beschrieben. Es wird die numerische Lösung der Dispersionsgleichung erhalten, das Anwachsen der Raumladungsweilen wird berechnet und seine Abhängigkeit von der Frequenz tendierend zur Frequenz des 2D-Plasmons wird untersucht.

\section{Introduction}

The theoretical study of collective plasma-beam interactions has begun from the pioneering works of Akhiezer and Fainberg [1] and Bohm and Gross [2]. Since that time these investigations have continued developing and now they form a separate branch in plasma physics, namely plasma electronics. Its achievements were the basis for the development of some investigation techniques, devices, and technologies [3, 4].

It is well known that the electron plasma devices have essential advantages compared to the vacuum electron devices, which are: (i) the possibility to vary the frequency band, (ii) to amplify and generate the electromagnetic waves in the millimeter and submillimeter region of the spectrum, (iii) a considerable enhancement of radiation power due to much greater values of current. Nevertheless, in the realization of such devices there are also some difficulties the main of which is the unstable operation of the plasma-beam devices, which in turn is the consequence of the space and time instability of the gaseous plasma itself.

Unlike the gaseous plasma, the solid state plasma is absolutely stable. For that reason, the local plasma instabilities in hot electron plasmas of semiconductors are probably

1) Zhodinskaya 4, 220141 Minsk, Republic of Belarus. 
the most promising basis for the creation of radiation sources and amplifiers in the submillimeter and far-infrared spectral regions. However, unlike beam instabilities in gaseous plasmas, they have not received similar attention in solid state plasma. In our opinion, the main reason for the lack of experimental studies of this effect in solid state systems so far is the difficulty in realizing appropriate electron beams in a semiconductor. In some of the previous works [5 to 7] the space-charge wave amplification induced by the beam instability in a semiconductor plasma of a multielectrode metal-insulatorsemiconductor microstructure was considered. There it was employed substantially that the electron distribution function of GaAs is inverted in a strong electric field of about $100 \mathrm{kV} \mathrm{cm}^{-1}$.

Such strong electric field causes some problems and for that reason in [8] we have proposed to use for the space-charge wave amplification an electron beam injected out of the quantum well into a 3D semiconductor plasma. The substantial improvement in epitaxial crystal growth during recent years has given a powerful tool for manufacturing semiconductor heterostructures with parallel layers. It is quite natural that the carriers ir such systems exhibit properties of a two-dimensional electron gas (2DEG) in the plane parallel to the layers. Having in mind further miniaturization of the amplifiers considered in [8], we can substitute the 3D electron plasma by 2DEG. Also it is essential that in [8] an amplification of the induced vibrations with frequencies less than the Langmuir frequency of the 3D electron plasma was considered. In other words, such instability could be called nonresonant. If we replace the three-dimensional plasma by 2DEG, the frequency of the induced vibrations tends to the plasma frequency, because the $2 \mathrm{D}$ plasmon frequency can be less than that of the $3 \mathrm{D}$ plasmon and near to the frequency band considered in [6]. Indeed, the estimates show that for the effective mass $m^{*} \approx 0.067 m_{\mathrm{e}}$ (GaAs, $m_{\mathrm{e}}$ is the free electron mass) and electron concentration $n \approx 10^{16} \mathrm{~cm}^{-3}$ the plasmon frequency $\omega_{\mathrm{p}, 3 \mathrm{D}}=\left(\pi n e^{2} / \mathrm{m}^{*}\right)^{1 / 2} \approx 2.8 \times 10^{13} \mathrm{~Hz}$. As for $2 \mathrm{D}$ plasmons, we should distinguish two cases: the first one is the thick insulator when $\omega_{\mathrm{p}, 2 \mathrm{D}}=\left(2 \pi N_{\mathrm{s}} e^{2} q / x m^{*}\right)^{1 / 2}$, and the second one the thin insulator when $\omega_{\mathrm{p}, 2 \mathrm{D}}=q\left(4 \pi N_{\mathrm{s}} e^{2} d_{\mathrm{in}} / \varkappa_{\mathrm{in}} m^{*}\right)^{1 / 2}$. Here $\varkappa=\varkappa_{\mathrm{sc}} \operatorname{coth} q d_{\mathrm{sc}}+\varkappa_{\mathrm{in}} \operatorname{coth} q d_{\mathrm{in}} . \varkappa_{\mathrm{sc}}$ and $\varkappa_{\mathrm{in}}$ are the static dielectric constants of the semiconductor and insulator, respectively, $N_{\mathrm{s}}$ is the surface electron density, $q$ the wave vector of the $2 \mathrm{D}$ plasmon, $d_{\mathrm{sc}}$ and $d_{\mathrm{in}}$ are the semiconductor and dielectric thicknesses. Supposing $N_{\mathrm{s}}$ to be $\approx 2 \times 10^{12} \mathrm{~cm}^{-2}$, $q \approx 2 \pi \times 10^{4} \mathrm{~cm}^{-1}, \quad d_{\mathrm{in}} \approx 10^{-5} \mathrm{~cm}, \quad d_{\mathrm{sc}} \approx 3 \times 10^{-6} \mathrm{~cm}, \quad \varkappa_{\mathrm{sc}}=12.5, \quad \varkappa_{\mathrm{in}}=3.9$, we have $\omega_{\mathrm{p}, 2 \mathrm{D}} \approx 3 \times 10^{12} \mathrm{~Hz}$ for the first case and if $d_{\mathrm{in}} \approx 3 \times 10^{-6} \mathrm{~cm}$, we have $\omega_{\mathrm{p}, 2 \mathrm{D}} \approx 10^{13} \mathrm{~Hz}$ for the second one.

So, this paper deals with the near-resonant amplification of a space-charge wave in $2 \mathrm{D}$ electron gas under electron beam injected out of the QW.

\section{Model Description and Main Equations}

As a rule, space-charge waves (SCW) in conducting media (in semiconductors, in particular) are understood as wave processes in a plasma of charge carriers with phase velocity close to the drift velocity. That means, solutions of the Maxwell equations exist which correspond to the slow waves. The main dynamical variables characterizing such quasistatic waves in a plasma of charge carriers in the absence of a magnetic field are the charge density and the potential. The well-known example of such SCW is the wave in the Gunn effect traveling through the wave amplifier [9]. From the electromagnetic 


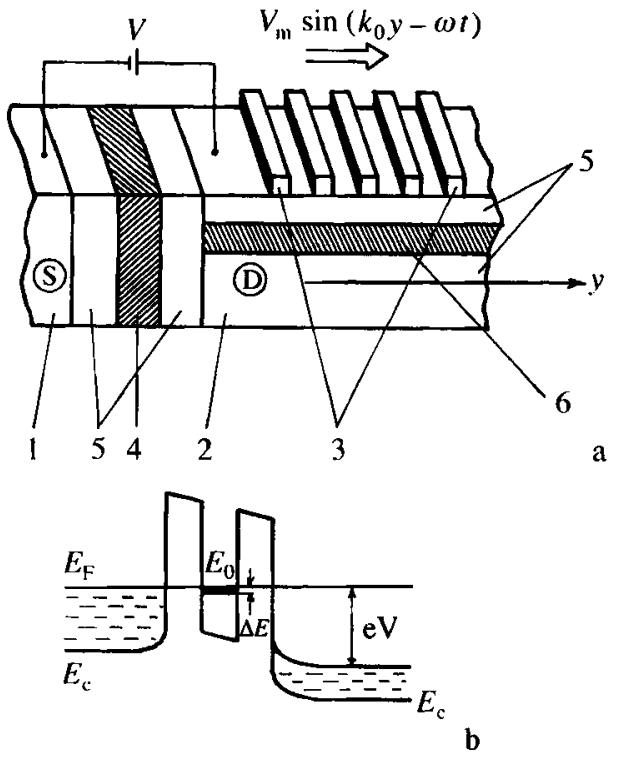

Fig. 1. a) Schematic representation of the semiconductor heterostructure in which the beam instability is induced: 1 source, 2 drain, 3 grating, $4 \mathrm{GaAs}, 5 \mathrm{Al}_{x} \mathrm{Ga}_{1-x} \mathrm{As}, V$ is the applied voltage, 6 the layer with the 2DEG. b) Energy diagram of the heterostructure (when the voltage $V$ is applied between source and drain). $E_{\mathrm{F}}$ is the Fermi level, $E_{0}$ the quantization level in the $\mathrm{QW}, E_{c}$ the bottom of the conduction band

point of view such SCW is some mode in the conducting medium. Another type of charge movement (in the form of some charge package) are, in a sense, the induced vibrations of charge density in microstructures called charge transfer devices [10] or charge coupled devices [11]. The controlled displacement of charge packages is caused by applying step-like voltage pulses to such structures. So, we shall consider SCW similar to the induced vibrations of charge density in CTD.

Let us consider the microstructure schematically represented in Fig. 1 a.

It is similar to that considered in [8] but differs from it in some respect. It is composed of the GaAs/AlGaAs heterostructure which forms the QW and the adjusted microstructure making the structure with $2 \mathrm{DEG}$ and additional grating on the surface. Suppose that a small potential of the form $V_{1}=V_{\mathrm{m}} \sin \left(k_{0} y-\omega t\right)$ is applied to the grating, where $k_{0}=2 \pi / l$ and $l$ is the spacing. Let us suppose further that a voltage $V_{2}$ is applied between the two domains of the structure which are labelled as source (S) and drain (D) and let the voltage be sufficient for the Fermi level to intersect the quantization level in the QW (Fig. 1b). Then the tunnel current which can be considered as the injected electrons in the drain, will flow through the microstructure. The electron velocity spread $\Delta v$ will be very small and correspond to the width of the quantization level $\Delta E$ which is approximately $1 \mathrm{meV}$.

The main equations which describe the physical situation in this case are the same as in [8], but for the sake of consistency and clearness we add here some comments concerning the treatment of electronic properties of a plasma in terms of hydrodynamic models.

To describe the vibrational processes in a plasma (including solid state plasma) it is possible to use the Boltzmann kinetic equation together with Maxwell equations. This method, however, is too complicated and in some cases is unnecessary. Indeed, the condition $\omega \tau \ll 1$, where $\tau$ is the mean relaxation time, implies the transition to the limit $\tau \rightarrow 0$, which in turn leads to the limit $l \rightarrow 0$, where $l$ is the mean free path. 
However, in this case, apart from its corpuscular structure, a plasma can be considered as a continuous medium. For that reason, there is no need to treat a plasma by means of particle distribution functions and it is sufficient to use a simplified hydrodynamic description where the main notions are the hydrodynamic velocity of the medium, its density, and pressure. All these quantities, they can be called macroscopic, can change in space and time but their changes are slow. That means the conditions $T_{\mathrm{m}} \gg \tau, L_{\mathrm{m}} \gg l$ are valid, where $T_{\mathrm{m}}$ and $L_{\mathrm{m}}$ are the characteristic time and length related to the essential changes of the corresponding macroscopic quantities.

It is reasonable to use the hydrodynamic description when the local quasiequilibrium particle distribution becomes settled fast. In our case it is convenient to distinguish three relaxation times: $\tau_{\mathrm{cc}}$, the plasma electron (or 2DEG) relaxation time, $\tau_{0 \mathrm{~b}}$, the beam electron relaxation time, and $\tau_{e b}$, the mean time for establishing equilibrium between them. If $\omega \tau \ll 1, \omega \tau_{0 \mathrm{~b}} \ll 1, \omega \tau_{\mathrm{cb}} \gg 1$, it is possible to treat the plasma as a two-component fluid.

An obvious estimate shows that the unperturbed velocity of the electrons injected into the 2DEG is greater than the thermal electron velocity. So, the electrons of the beam and of 2DEG could be considered as two reciprocally penetrating fluids which can be treated in terms of hydrodynamic equations together with the Poisson equation. In the absence of an external magnetic field they are [12]

$$
\begin{aligned}
& \frac{\partial \mathbf{v}_{1 j}}{\partial t}+\left(\mathbf{v}_{1 j} \nabla\right) \mathbf{v}_{1 j}=\frac{e}{m_{j}^{*}} \nabla \boldsymbol{\Phi}-\frac{\nabla p_{j}}{m_{j}^{*} n_{1 j}}-v_{j}\left(\mathbf{v}_{1 j}-\mathbf{v}_{0 j}\right), \\
& \frac{\partial n_{1 j}}{\partial t}+\operatorname{div}\left(n_{1 j} \mathbf{v}_{1 j}\right)=0 \\
& \operatorname{div} \mathbf{E}=-\nabla \boldsymbol{\Phi}=-4 \pi e \sum_{j}\left(n_{1 j}-n_{0 j}\right) .
\end{aligned}
$$

Here $\mathbf{v}_{1 j}$ and $n_{1 j}$ are the hydrodynamic velocity and the concentration of the particles of $j$-th kind, respectively, subscript $j$ becomes equal to e (electrons of $2 \mathrm{DEG}$ ) or b (beam electrons); $n_{0 j}$ is the equilibrium concentration, $p_{j}$ the pressure, $\Phi$ the potential, and $\mathbf{E}$ the electric field of an external perturbation: $\mathbf{E}=-\nabla \Phi ; \nu_{j}$ is the collision frequency.

As usual, we believe that the charge of the 2DEG electrons and beam electrons is compensated by the positive charge of the lattice ions. We also believe that

$$
n_{1 j}=n_{0 j}+n_{j}, \quad \mathbf{v}_{1 j}=\mathbf{v}_{0 j}+\mathbf{v}_{j}, \quad n_{j} \ll n_{0 j}, \quad v_{j} \ll v_{0 j},
$$

where $n_{j}$ and $v_{j}$ are the deviations from the corresponding equilibrium values.

Supposing the pressure to be linearly dependent on the temperature $\left(p_{j}=T_{j} n_{j}\right.$, where $T_{j}$ is the temperature of the particles of $j$-th kind) and neglecting the terms which consist of the products of small factors, we obtain the following system of linear equations:

$$
\begin{aligned}
& \frac{\partial \mathbf{v}_{j}}{\partial t}+\left(\mathbf{v}_{0 j} \nabla\right) \mathbf{v}_{j}=-\frac{e}{m_{j}^{*}} \mathbf{E}-\frac{v_{T j}^{2}}{n_{0 j}} \nabla n_{j}-v_{j} \mathbf{v}_{j}, \\
& \frac{\partial n_{j}}{\partial t}+\operatorname{div}\left(n_{0 j} \mathbf{v}_{j}+n_{j} \mathbf{v}_{0 j}\right)=\mathbf{0}, \\
& \nabla \Phi=4 \pi e \sum_{j} n_{j},
\end{aligned}
$$

where we also introduce the notation $v_{T j}=\left(2 k_{\mathrm{B}} T_{j} / m_{j}^{*}\right)^{1 / 2}$. 
Having used the standard procedure (see $[8,13]$ ), we have from $(3)$ the following dispersion equation for collective vibrations in the $2 \mathrm{DEG}$ with the beam:

$$
\begin{aligned}
& 1-\frac{\Omega_{\mathrm{e}}^{2}}{\omega\left(\omega+i v_{\mathrm{e}}\right)}-\frac{\Omega_{\mathrm{b}}^{2}}{\left(\omega-k_{0} v_{0 \mathrm{~b}}\right)^{2}}-\frac{k^{2} v_{T \mathrm{e}}^{2}}{\omega^{2}}=0, \\
& \Omega_{j}=\left(\frac{4 \pi e^{2} n_{0 j}}{\varepsilon(\mathbf{q}, \omega) m_{j}^{*}}\right)^{1 / 2} ; \quad j=\mathrm{e}, \mathrm{b} .
\end{aligned}
$$

Here we also believe that the hydrodynamic velocity of the 2DEG electrons is $v_{0 \mathrm{c}}=0$, the unperturbed velocity of the beam electrons $v_{0 \mathrm{~b}}$ is directed along the $y$-axis and the particle collisions in the beam can be neglected.

However, in comparison with the corresponding dispersion equation of [8], here there is an essential difference: $\varepsilon$ is no longer the static dielectric constant of the semiconductor but a nonlocal function depending on the frequency $\omega$ and the wave vector $q$ of the plasmon. For the 2DEG it can be expressed in a simple form when the inversion layer is a sheet of charge in the plane embedded in a homogeneous medium with dielectric constant $\varepsilon_{0}$. Then one can define the dielectric function for longitudinal excitation of the 2DEG as [14]: $\varepsilon(\mathbf{q}, \omega)=\varepsilon_{0}+2 \pi \beta(\mathbf{q}, \omega) \chi(\mathbf{q}, \omega)$, where $\beta(\mathbf{q}, \omega)=\left(q^{2}-\varepsilon_{0} \omega^{2} / c^{2}\right)^{1 / 2}$ and the polarizability $\chi(q, \omega)=\chi_{1}+i \chi_{2}$ is of the form

$$
\begin{aligned}
& \chi_{1}=G\left\{2 z-C_{-}\left[(z-u)^{2}-1\right]^{1 / 2}-C_{+}\left[(z+u)^{2}-1\right]^{1 / 2}\right\}, \\
& \chi_{2}=G\left\{D_{-}\left[1-(z-u)^{2}\right]^{1 / 2}-D_{+}\left[1-(z+u)^{2}\right]^{1 / 2}\right\} \\
& G=\frac{2 m^{*} e^{2} N_{\mathrm{s}}}{\hbar^{2} q^{3} k_{\mathrm{F}}}, \quad z=\frac{q}{2 k_{\mathrm{F}}}, \quad u=\frac{\omega}{q v_{\mathrm{F}}} .
\end{aligned}
$$

Here $N_{\mathrm{s}}$ is the number of electrons per unit area, $k_{\mathrm{F}}$ and $v_{\mathrm{F}}$ are the Fermi wave vector and the Fermi velocity, respectively; $C_{ \pm}=\operatorname{sgn}(z \pm u)$ and $D_{ \pm}=0$ for $|z \pm u|>1$; $C_{ \pm}=0$ and $D_{ \pm}=1$ for $|z \pm u|<1$.

\section{Increment of the Space-Charge Wave}

Let the frequency $\omega$ be complex and equal to $\omega=q v_{0}+\Delta+i \gamma$, where $q v_{0}=\omega_{0}$ is the frequency corresponding to the exact phase resonance, $q$ is the wave number of the space-charge wave which in our case is equal to the spacing of the grating $\left(q=k_{0}\right), \Delta$ is the detuning, and $\gamma$ the increment. Here we omitted the subscript b at the beam velocity. Separating in (5) the real and imaginary parts and introducing the dimensionless detuning $x=\Delta / \omega_{0}$ and the increment $y=\gamma / \omega_{0}$ we obtain the system of equations

$$
\begin{aligned}
& m(x, y) n(x, y) p(x, y)-\left(c_{1} /|\varepsilon|^{2}\right)\left[\operatorname{Re} \varepsilon^{*} \operatorname{Re} s(x, y)+\operatorname{Im} \varepsilon^{*} s(x, y)\right]-\left(c_{2} /|\varepsilon|^{2}\right) \\
& \times\left[\operatorname{Re} \varepsilon^{*} \operatorname{Re} t(x, y)+\operatorname{Im} \varepsilon^{*} \operatorname{Im} t(x, y)\right]-\lambda_{1} \operatorname{Re} w(x, y)=0, \\
& \left(c_{1} /|\varepsilon|^{2}\right)\left[\operatorname{Re} \varepsilon^{*} \operatorname{Im} s(x, y)-\operatorname{Im} \varepsilon^{*} \operatorname{Re} s(x, y)\right]+\left(c_{2} /|\varepsilon|^{2}\right) \\
& \times\left[\operatorname{Re} \varepsilon^{*} \operatorname{Im} t(x, y)-\operatorname{Im} \varepsilon^{*} \operatorname{Re} t(x, y)\right]+\lambda_{1} \operatorname{Im} w(x, y)=0 .
\end{aligned}
$$

Here $m(x, y), n(x, y), p(x, y)$ are the polynomials with real coefficients while $s(x, y)$, $t(x, y), w(x, y)$ are polynomials with imaginary ones. For the readers' convenience we 

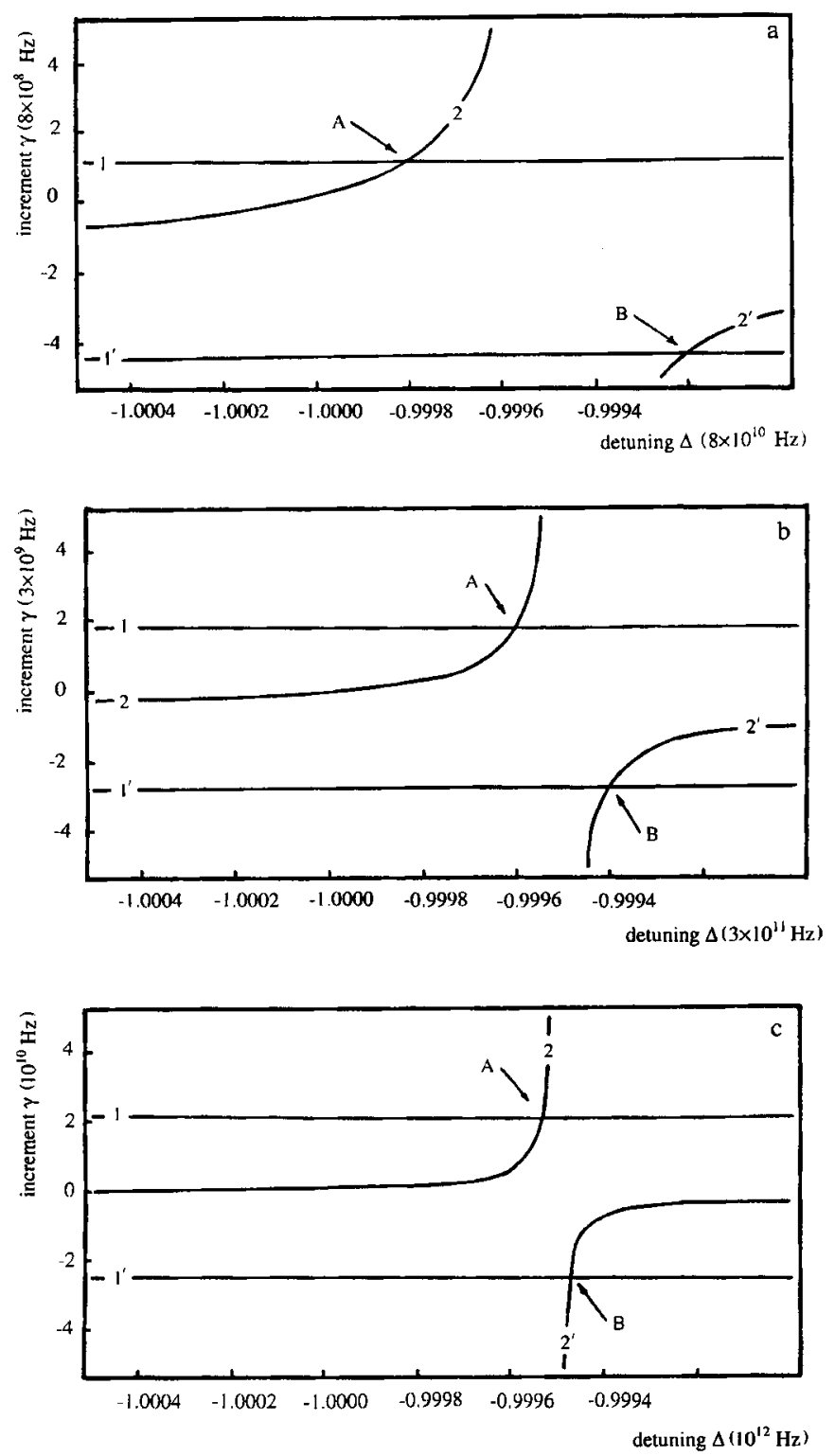

Fig. 2. The numerical solution of the system (7) (see the text). The coordinates of the two points marked by the letters $\mathrm{A}$ and $\mathrm{B}$ correspond to the detuning and increment of the two waves, respectively. The first wave is amplified (A) while the other is damped (B). The assumptions approved by the computations are the following: $l=10^{-4} \mathrm{~cm}, T_{\mathrm{c}}=50 \mathrm{~K}, N_{\mathrm{s}}=2 \times 10^{11} \mathrm{~cm}^{-2}, n_{0 \mathrm{e}}=10^{16} \mathrm{~cm}^{-3}$, $n_{0 \mathrm{~b}}=5 \times 10^{12} \mathrm{~cm}^{-3}$, a) $\left.\omega_{0}=8 \times 10^{10} \mathrm{~Hz}, \mathrm{~b}\right) \omega_{0}=3 \times 10^{11} \mathrm{~Hz}(\mu<1)$, c) $\omega_{0}=10^{12} \mathrm{~Hz}(\mu>1)$

add all the most tiresome formulae in the Appendix. The asterisk stands for the complex conjugate, $c_{1}=4 \pi e^{2} n_{0 \mathrm{e}} / m^{*} \omega_{0}^{2}, c_{2}=4 \pi e^{2} n_{0 \mathrm{~b}} / m^{*} \omega_{0}^{2}, \lambda_{1}=v_{T_{\mathrm{c}}}^{2} / v_{0}^{2}$.

Also we have noticed and took into account the condition $q^{2}=k_{0}^{2} \gg \varepsilon_{0} \omega_{0}^{2} / c^{2}$, which has lead to a considerable simplification of formulae (6) (for details see the Appendix). 
Each of the equations (7) defines some curve $f_{n}(x, y)=0(n=1,2)$ on the $(x, y)$ plane and, therefore, if two curves intersect in some point $\mathrm{P}$ with coordinates $\left(x_{0}, y_{0}\right)$, this pair of numbers satisfies the system (7). The numerical solution of (7) is achieved by the special program plotting $f_{n}$ for some $x \in\left[x_{\mathrm{in}}, x_{\mathrm{fill}}\right]$; for this purpose another program was used which calculated the zeroes of $f_{n}\left(x_{1}, y\right)$ at the point $x_{1} \in\left[x_{\mathrm{in}}, y_{\mathrm{fin}}\right]$ on some interval $\left[y_{\text {in }}, y_{\text {fin }}\right]$.

The following assumptions were made: the effective masses of the beam electrons $m_{\mathrm{b}}^{*}$ and the 2DEG electrons $m_{\mathrm{e}}^{*}$ were believed to be equal to each other $\left(0.067 m_{0}\right.$, where $m_{0}$ is the free electron mass), the collision frequency $v_{\mathrm{e}}$ was believed to be equal to the reciprocal momentum relaxation time. The latter was extracted from the experimental data for the Hall mobility in a 2DEG [15]. The spacing of the grating was believed to be equal to $l=10^{-4} \mathrm{~cm}$, and the ratio $n_{0 \mathrm{~b}} / n_{0 \mathrm{e}} \approx 10^{-5}$. The most interesting results are summarized in Fig. 2a and c. The curves labelled 1 and $1^{\prime}$ in Fig. $2 a, c$ are the loci of zeroes of $(7 \mathrm{a})$, while the other labelled 2 and $2^{\prime}$ are the loci of zeroes of $(7 \mathrm{~b})$. The most remarkable feature of Fig. 2 is the two points of intersection of curves $1,1^{\prime}$ and $2,2^{\prime}$.
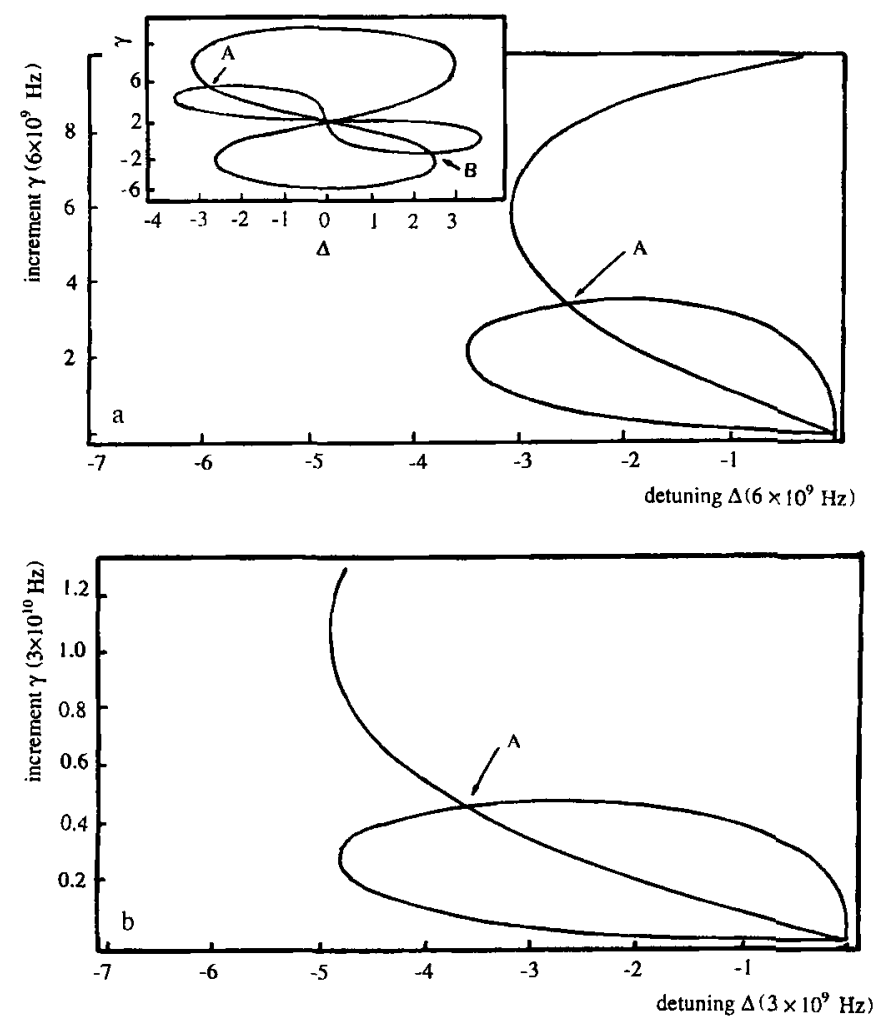

Fig. 3. The numerical solution of the dispersion equation of [6]. In the inset of Fig. 3a we represent on a reduced scale both branches of the solution. The two points labelled by the letters $\mathrm{A}$ and $\mathrm{B}$ correspond to two waves one of which is amplified (A) while the other is damped (B). Having scaled up, we represented in Fig. 3a, b only one of them (A). The parameters approved by the calculations are: $n_{0 \mathrm{e}}=10^{16} \mathrm{~cm}^{-3}, n_{0 \mathrm{~b}}=5 \times 10^{12} \mathrm{~cm}^{-3}, l=10^{-4} \mathrm{~cm}, T_{\mathrm{e}}=50 \mathrm{~K}$, a) $\omega_{0}=6 \times 10^{11} \mathrm{~Hz}$, b) $\omega_{0}=3 \times 10^{11} \mathrm{~Hz}$ 
From the mathematical point of view this is the direct consequence of the evenness of the polynomials of (7). From the physical point of view these two points correspond to two waves spreading in 2DEG: the first one has a positive increment and is amplifying while the other has a negative increment and is damping. Such phenomenon is well known for the gaseous plasma and, moreover, if the collisions in a gaseous plasma are neglected, the frequencies of these two waves can be expressed explicitly as $\omega_{\mp}=k_{0} v_{0} \mp \Omega_{\mathrm{b}} / \sqrt{\varepsilon\left(k v_{0}\right)}$, and besides, the wave with frequency $\omega_{-}$amplifies while the other one with $\omega_{+}$is damped.

These two waves exist also in case of an electron beam injected out of the QW into a $3 \mathrm{D}$ plasma. To compare these results we represent the corresponding solutions of the dispersion equation of [8] in Fig. 3a, b. It is also worth to note that in case of a 2DEG it is natural to expect an increase of the increment if the frequency of space-charge vibrations tends to the 2D plasmon frequency, because the increments of the resonant instabilities are always greater than the nonresonant ones. As can easily be seen from Fig. $2 \mathrm{a}, \mathrm{c}$, indeed, if the frequency $\omega_{0}$ tends to the frequency of self-vibrations (to the frequency of the $2 \mathrm{D}$ plasmon) the increment gradually increases. Unfortunately, the absolute value of the increment in case of electron injection into a 2DEG cannot be greater than in the case of a $3 \mathrm{D}$ plasma, because if the frequency of the space-charge wave tends to the frequency of the $2 \mathrm{D}$ plasmon, the detuning of the two waves $\omega_{-}$and $\omega_{+}$gradually becomes smaller, until the two waves merge.

\section{Discussion}

As was stated in the Introduction, the creation of coherent sources of radiation and amplifiers in submillimeter and far-infrared regions is one of the keen problems of solid state electronics, because by now there are still no suitable amplifiers and generators for some frequency bands. In particular, it is important to have active receiving and transmitting devices for atmospheric windows, i.e. for the regions of $35,95,140,200$, and $500 \mathrm{GHz}$.

Here we have discussed the possibility of space-charge wave amplification in a two-dimensional electron gas of an (AlGa)As-GaAs heterostructure with grating. The injection of electrons out of the quantum well by means of resonant tunneling leads to beam instability and amplification of the space-charge wave. Elementary effects of radiation by the beam particles are the only mechanisms which provide transmission of kinetic energy from the beam to the wave. The necessary condition for that is $\omega \approx k_{0} V_{0 \mathrm{~b}}$. The amplification mechanism is the resonant energy exchange between the wave and the particles of the beam which gives rise to an increase of the wave amplitude. Since for $\omega<\Omega_{\mathrm{c}}$ the dielectric constant is negative, it provides not a repulsion but an attraction of the negative charges of the beam and their further bunching due to the initially induced or spontaneously emerging weak modulation. Thus, the condition $\omega \approx k_{0} V_{0 \mathrm{~b}}$ defines the distance between the bunches [16]. Since the velocity of the beam electrons is greater than the phase velocity of the wave, the space charge will radiate just as free electrons due to the Cherenkov effect, and the local bunches will play the role of 'Cherenkov emitters'.

If the frequency $\omega$ tends to the frequency of self-vibrations (to the frequency of the two-dimensional plasmons), the increment of SCW increases, but the resonant and nonresonant instabilities transform one into another continuously and differ only by the increment value. It is also clear that the frequency of the gate field cannot exceed some definite value restricted by the spatial ability of SCW to follow the field. Such limit 
might be determined by the reciprocal Maxwell relaxation time $\tau_{M}^{-1}$ and does not exceed approximately $10^{12} \mathrm{~Hz}$ at a chosen value of electron concentration.

In our opinion, the structure proposed in this paper could be the basis for working out a new type of solid state amplifiers for the frequency band up to $500 \mathrm{GHz}$. As for the criterion of the transition from convective instability to the absolute one, and hence, for the possibility to work out a generator on this basis, the problem should be investigated further. The work is under progress now and the results will be presented later. Also the problem how to lead the radiation out of the sample is beyond the scope of this paper. Perhaps, the appropriate way for this is to use some waveguide for the surface electromagnetic waves, but this is no more than a working hypothesis and the problem should be considered separately.

\section{Appendix}

The polynomials of equations (7) are of the form

$$
\begin{aligned}
& m(x, y)=\left[\left(1+x^{2}\right)^{2}+\left(y+\lambda_{2}\right)^{2}\right]\left[(1+x)^{2}+y^{2}\right] \\
& n(x, y)=\left(x^{2}-y^{2}\right)^{2}, \quad p(x, y)=\left[(1+x)^{2}-y^{2}\right]^{2}, \\
& \operatorname{Re} s(x, y)=(1+x)^{2}-y\left(y+\lambda_{2}\right), \quad \operatorname{Im} s(x, y)=(1+x)\left(2 y+\lambda_{2}\right), \\
& \operatorname{Re} t(x, y)=x^{2}-y^{2}, \quad \operatorname{Im} t(x, y)=2 x y, \\
& \operatorname{Re} w(x, y)=(1+x)^{2}-y^{2}, \quad \operatorname{Im} w(x, y)=2 y(1+x),
\end{aligned}
$$

where $\lambda_{2}=\nu_{\mathrm{c}} / \omega_{0}$.

Taking into account the condition $q^{2}=k^{2} \gg \varepsilon_{0} \omega_{0}^{2} / c^{2}$ and introducing the parameter $\mu=\left|k_{0} / 2 k_{\mathrm{F}} \pm \omega_{0} m^{*} / \hbar k_{0} k_{\mathrm{F}}\right|$, we have for the dielectric function of the 2DEG the following expressions:

(i) $\mu<1$ :

$$
\begin{aligned}
& \operatorname{Re} \varepsilon^{*}=\varepsilon_{0}+2 \pi k_{0}^{2} G / k_{\mathrm{F}}+2 \pi k_{0} G\left[\sqrt{r_{2}} \cos \left(\varphi_{2} / 2\right)-\sqrt{r_{1}} \cos \left(\varphi_{1} / 2\right)\right] \\
& \operatorname{Im} \varepsilon^{*}=2 \pi k_{0} G\left[\sqrt{r_{1}} \sin \left(\varphi_{1} / 2\right)-\sqrt{r_{2}} \sin \left(\varphi_{2} / 2\right]\right] \\
& r_{1}=\sqrt{\left(1-\alpha_{1}^{2}(x)+\delta^{2}(y)\right)^{2}+4 \alpha_{1}^{2}(x) \delta^{2}(y)}, \quad \varphi_{1}=\operatorname{arctg} \frac{2 \alpha_{1}(x) \delta(y)}{1+\delta^{2}(y)-\alpha_{1}^{2}(x)},
\end{aligned}
$$

where

$$
\begin{aligned}
& r_{2}=\sqrt{\left(1-\alpha_{2}^{2}(x)+\delta^{2}(y)\right)^{2}+4 \alpha_{2}^{2}(x) \delta^{2}(y)}, \quad \varphi_{2}=\operatorname{arctg}-\frac{2 \alpha_{2}(x) \delta(y)}{1+\delta^{2}(y)-\alpha_{2}^{2}(x)} \\
& \alpha_{1}(x)=\frac{q}{2 k_{\mathrm{F}}}-\frac{\omega_{0}(1+x)}{q v_{\mathrm{F}}}, \quad \delta(y)=\frac{\omega_{0} y}{q v_{\mathrm{F}}}, \quad \alpha_{2}(x)=\frac{q}{2 k_{\mathrm{F}}}+\frac{\omega_{0}(1+x)}{q v_{\mathrm{F}}}
\end{aligned}
$$

(ii) $\boldsymbol{\mu}>1$ :

In this case $r_{1}$ and $r_{2}$ in (A1), (A2) should be replaced by $r_{1}^{\prime}, r_{2}^{\prime}$ which are of the form

$$
\begin{aligned}
& r_{1}^{\prime}=\sqrt{\left(\alpha_{1}^{2}(x)-\delta^{2}(y)-1\right)^{2}+4 \alpha_{1}^{2} \delta^{2}(y)}, \\
& r_{2}^{\prime}=\sqrt{\left(\alpha_{2}^{2}(x)-\delta^{2}(y)-1\right)^{2}+4 \alpha_{2}^{2} \delta^{2}(y)} .
\end{aligned}
$$


Acknowledgement The authors are greatly indebted to the Fund for Fundamental Research, Republic of Belarus, for financial support (Grant No. 006-012).

\section{References}

[1] A. I. Akhiezer and Ya. B. Fainberg, Dokl. Akad. Nauk SSSR 69, 555 (1949).

[2] D. BoHm and E. P. Gross, Phys. Rev. 75, 1851 (1949).

[3] R. J. Briggs, Electron-Stream Interaction with Plasma, Cambridge MIT Press, 1964.

[4] G. A. Bernashevskil, E. V. Bogdanov, V. Ya. Kislov, and Z. S. Chernov, Plasma and Electron Amplifiers and Generators of SHF, Izd. Sovetskoe Radio, Moscow 1965 (in Russian).

[5] I. E. Tratle and A. B. Filonov, phys. stat. sol. (b) 173, 775 (1992).

[6] I. E. Tralle and A. B. Fllonov, phys. stat. sol. (b) 182, 165 (1994).

[7] I. E. Trailie and A. B. Filonov, J. Phys. D 27, 1707 (1994).

[8] I. E. Tralue and V. A. Siz.Juk, phys. stat. sol. (b) 182, 171 (1994).

[9] R. H. Dean, A. B. Drefben, J. F. Kaminski, and A. Triano, Electronics Letters 6, 775 (1970).

[10] C. Sequin and M. Tompset, Charge Transfer Devices, Academic Press, New York 1975 (p. 8).

[11] D. F. Barise (Ed.), Charge-Coupled Devices, Springer-Verlag, Berlin 1980 (p. 6).

[12] V. L. Ginzsuurg and A. A. Ruchadze, The Waves in Magnetoactive Plasma, Izd. Nauka, Moscow 1975 .

R. S. Sagidefe, M. Rosenbluth, A. A. Galeev, and R. Sudan, Principles of Plasma Physics, Vol. 1, Izd. Energoatomizdat, Moscow 1983 (Soviet-American ed.).

[13] L. A. Ar'tsimovich and R. S. Sagdeev, Plasma Physics for Physicists, Izd. Atomizdat, Moscow 1979 (in Russian).

[14] F. Stern, Phys. Rev. Letters 18, 546 (1967).

[15] T. ANido, A. Fowler, and F. Stern, Rev. mod. Phys. 54, 437 (1982).

[16] A. N. Kondratenko and V. M. Kuklin, Principles of Plasma Electronics, Izd. Energoatomizdat, Moscow, 1988 (in Russian). 